\title{
Correlations among Pulmonary DJ-1, VDR and Nrf-2 in patients with Chronic Obstructive Pulmonary Disease: A Case-control Study
}

\author{
Ying Xiang ${ }^{1,2^{*}}$, Lin Fu ${ }^{1,2 \llbracket *}$, Hui-Xian Xiang1, Ling Zheng1, Zhu-Xia Tan ${ }^{1,2}$, Li-Xiang Wang1, Wei Cao1, \\ De-Xiang $\mathrm{Xu}^{2}$ and Hui Zhao ${ }^{1,2}$ \\ 1. Respiratory and critical care medicine, Second Affiliated Hospital of Anhui Medical University, Hefei, 230601, China. \\ 2. Department of Toxicology, Anhui Medical University, Hefei, 230032, China. \\ *These authors contributed equally to this work. \\ $\triangle$ Corresponding authors: Prof. Lin Fu, Second Affiliated Hospital, Anhui Medical University, Hefei 230022, China. E-mail: fulindev@126.com; Prof. Hui Zhao, Second \\ Affiliated Hospital, Anhui Medical University, Hefei 230022, China. E-mail: zhaohuichenxi@126.com; Prof. De-Xiang Xu, Department of Toxicology, Anhui Medical
} University, Hefei 230032, China. E-mail: xudex@126.com; Tel: +86551 65167923.

(C) The author(s). This is an open access article distributed under the terms of the Creative Commons Attribution License (https://creativecommons.org/licenses/by/4.0/). See http://ivyspring.com/terms for full terms and conditions.

Received: 2021.01.20; Accepted: 2021.04.02; Published: 2021.04.22

\begin{abstract}
Parkinson protein 7 (PARK7)/DJ-1 (DJ-1) is a redox sensitive molecular and stabilizer of nuclear factor erythroid 2-related factor 2 (Nrf-2). Nrf-2 regulates the downstream antioxidant defense system and exerts a significant function in patients with chronic obstructive pulmonary disease (COPD). Vitamin D receptor (VDR) is the nuclear receptor that regulates the downstream target genes. This study aimed to analyze the associations among pulmonary function, DJ-1, VDR and Nrf-2 in COPD patients. Serum was collected from 180 COPD patients and control subjects. Thirty-five lung tissues were obtained. DJ-1 was measured using ELISA and western blotting. Nrf-2 and VDR were detected by immunohistochemistry. Serum and pulmonary DJ-1 levels were lower in COPD patients than those in control subjects. Pulmonary VDR-positive nuclei were reduced in COPD patients. Nrf-2-positive nuclei were reduced in lung tissues of COPD patients. On the contrary, Nrf-2-related downstream target proteins were elevated in COPD patients. Further correlation analysis indicated that forced expiratory volume in 1 second (FEVI) was positively associated with pulmonary DJ-1, VDR and Nrf-2 in patients with COPD. In addition, there were positive correlations among DJ-1, VDR and Nrf-2 in lung tissues of COPD patients. In conclusion, DJ-1, VDR and Nrf-2 were decreased in COPD patients compared with control subjects. The reduction of DJ-1 and VDR associating with Nrf-2 downregulation may be involved in the process of COPD.
\end{abstract}

Key words: DJ-1; VDR; Nrf-2; lung; COPD; pulmonary function

\section{Introduction}

Chronic obstructive pulmonary disease (COPD), which accompanied with irreversible airflow limitation and chronic airway inflammation, is the third dominating reason of morbidity and mortality and result in tremendous economic pressures of health care all over the world [1]. The pathogenesis and pathology of COPD are complex, but excessive airway inflammation reaction, oxidative stress, imbalance between protease/antiprotease system and immunological mechanisms are the most common etiological factors [2]. Recent researches indicated that a noxious environmental insult could evoke the occurrence and development of
COPD. Cigarette smoking has been extensively regarded as the most important risk factor for COPD $[3,4]$. The management and treatment of cigarette smoking-caused COPD is still a huge challenge for all humanity.

Parkinson protein 7 (PARK7)/DJ-1 (DJ-1) was first identified as a gene mutated in a recessively inherited form of early-onset Parkinson disease $[5,6]$. DJ-1 is a sensitive molecular of oxidative stress with a reactive cysteine at position 106 (Cys106). Under oxidative stress, DJ-1 plays an important role in eliminating hydrogen peroxides, which is oxidized into cysteine sulfnate and sulfonate [7]. There is 
substantial evidence that over-expression of DJ-1 alleviated neurodegeneration caused by oxidative stress in rats [8]. Related to its anti-oxidant properties, DJ-1 can not only regulate cell survival and proliferation, but also suppress cell death signaling [9]. Additionally, it is reported that DJ-1-deficient mice easily suffered from oxidative stress-induced severe heart failure [10]. However, the change of pulmonary DJ-1 was unclear in COPD patients.

Nuclear factor erythroid 2-related factor 2 (Nrf-2) is a key transcription factor that regulates the downstream antioxidant defense system and exerts a significant function in several pulmonary diseases which relates oxidative stress and inflammation [11]. Research has found that DJ-1 inhibits Nrf-2 from combining with Kelch-like ECH-associated protein 1 (Keap-1) and attenuates the subsequent ubiquitination of Nrf-2, which is essential for maintaining the stability of the antioxidant enzymes under reactive oxygen species (ROS) stimulation [12, 13]. Moreover, Vitamin D and its receptor (VDR) exert a significant role in the metabolism and homeostasis of calcium and phosphate [14]. Recently, in vivo and in vitro experiments indicated that vitamin $\mathrm{D}$ supplementation could activate VDR and upregulate Nrf-2 expression in different cells [15-17]. However, the association among DJ-1, Nrf-2 and VDR was unclear in COPD patients. The aim of this study was to analyze the associations among DJ-1, Nrf-2 and VDR in lungs of COPD patients based on a case-control study. Our results found that DJ-1 was reduced in serum and lung tissues of COPD patients. Pulmonary VDR and Nrf-2 was decreased in COPD patients. Our data provide evidences that DJ-1 is positively associated with Nrf-2 and VDR in lung tissues of COPD patients.

\section{Materials and methods}

\section{Reagents}

DJ-1, HO-1, NOX-4, Nrf-2 and VDR antibodies were purchased from Cell Signaling Technology (MA, USA). Chemiluminescence (ECL) detection kits were from Advansta (CA, USA). All other chemicals were prepared from Sigma Chemical Co. (MO, USA) unless specifically noted.

\section{Participants' inclusion}

All COPD patients were randomly selected from Anhui COPD Cohort (AHCC) study which was a hospital-based prospective cohort study in Second Affiliated Hospital of Anhui Medical University in China between January 2017 and September 2019. For matched case-control study, patients with COPD were recruited. Pulmonary function was tested in all COPD patients. COPD was confirmed on basis of the
American Thoracic Society criteria and the Global Initiative for COPD (GOLD) criteria [1], which forced expiratory volume in $1 \mathrm{~s} /$ forced vital capacity ratio was less than 70\%. Moreover, all 180 sex-, age- and race-matched control subjects were randomly selected from the physical examination center of Second Affiliated Hospital. Serum was collected between COPD patients and control subjects. In order to evaluate the protein expressions of DJ-1 and oxidative stress markers, 35 human lung tissues of COPD patients were collected in surgery. Moreover, control subjects were paracancerous tissues, which were from histologically confirmed lung cancer patients without COPD. This study was approved by Ethics Committee of Anhui Medical University. All participants have agreed and signed an informed consent.

\section{Western blotting}

Western blotting was performed as the process of previous study [18]. Sixty mg lung tissue was added into in the EP tube. Pulmonary protein was extracted using RIPA buffer ( $500 \mu \mathrm{L}$ RIPA, $5 \mu \mathrm{L} 1 \%$ PMSF, $5 \mu \mathrm{L}$ phosphatase inhibitor). Lung tissues were homogenized ultrasonic treatment for 15 second on the ice. Lysates were separated through highspeed cryogenic centrifugal. Then, the middle layer was collected. Protein concentrations were detected through BCA protein assay kit. Total $15 \mu \mathrm{g}$ protein of each sample was added and separated in the $10 \%$ SDS-PAGE gel through electrophoresis. Then, protein was transferred to PVDF membranes. Membranes were blocked in no-fat milk for $3 \mathrm{~h}$ at room temperature. After, membranes were washed three times using distilled water. Then, primary antibodies including DJ-1 (1:2000), HO-1 (1:2000), NOX-4 (1:2000) were incubated overnight at $4{ }^{\circ} \mathrm{C}$ refrigerator, second antibodies of different dilution ratios were continued to incubate $2.5 \mathrm{~h}$ at $37^{\circ} \mathrm{C}$ incubator. At last, detection was visualized with ECL kit. Each protein was quantified after normalization to band of $\beta$-actin using densitometric analysis.

\section{Immunochemistry (IHC)}

Human lung tissues were fixed in formalin and embedded in paraffin. Pulmonary sections $(5 \mu \mathrm{m})$ were dewaxed and rehydrated according to another research [19]. To punch cell membrane and suppress endogenous peroxidase, sections were immersed in PBS containing $0.5 \%$ Triton X-100 and $3 \% \mathrm{H}_{2} \mathrm{O}_{2}$ for 45 min. Antigen retrieval was performed in boiled citrate solution. Then, the section was blocked in serum, VDR primary antibody (1:200) was incubated at $37^{\circ} \mathrm{C}$ incubator for $3.5 \mathrm{~h}$. After washed in PBS three times, conjunction with streptavidin-HRP complex was subsequent incubated for $2.5 \mathrm{~h}$ at room 
temperature. Immunolabelling was evaluated using DAB solution and nuclei were stained with hematoxylin in a dark room. VDR-positive nuclei were calculated in nine randomly selected fields, by two independent pathologists without prior knowledge of the experimental design.

\section{ELISA}

DJ-1 ELISA kits were come from Cusabio, Wuhan, China (https://www.cusabio.com/). Inflammatory cytokines (MCP-1, MIP-1 and TNF-a) were purchased from Wuhan ColorfulGene Biological Technology Co., Ltd. Serum DJ-1 and inflammatory cytokines were measured in a microplate reader using protocols as described previously [20].

\section{Statistical analysis}

Statistical analysis was carried out with SPSS 21.0. All data were expressed as means and medians. Independent sample unpaired $t$ test was evaluated the difference for continuous variables between two groups. Chi-square test was executed for count data between two groups. The correlations among DJ-1, VDR, Nrf-2 and FEV1 were analyzed using Spearman correlation analysis. $\quad P<0.05$ was considered statistically significant.

\section{Results}

\section{Demographic characteristics and clinical information}

Demographic characteristics and clinical information were represented in Table 1 . One hundred and eighty COPD patients (73.2\% males) and control subjects $(63.2 \%$ males) were recruited in this project. The median ages were 74.0 and 67.0 years in COPD patients and control subjects, respectively. Routine blood test was carried out. The results indicated that the counts of white blood cells (WBC), neutrophils and monocytes were increased in COPD patients. The number of lymphocytes was increased in COPD patients. Moreover, the levels of inflammatory cytokines in serum were detected in COPD patients and control subjects. We found that CRP, TNF- $\alpha$, IL-6 and MCP-1 were significantly elevated in COPD patients. Moreover, pulmonary function was measured in COPD patients. The median FVC, FEV1 and FEV1/FVC of current COPD patients was $1.88 \mathrm{~L}, 40.4 \%$ and $51.3 \%$, respectively (Table 1).

\section{DJ-1 in serum and lung tissues in COPD patients and control cases}

Serum DJ-1 was detected using ELISA in COPD patients and control subjects. We found that DJ-1 was reduced in COPD patients (Figure 1A). Moreover, the levels of serum DJ-1 were analyzed in different grades of COPD patients. The results indicated that serum DJ-1 was gradually decreased in parallel with the grades of COPD patients (Figure 1B). In addition, the expression of DJ-1 was detected in lung tissues of COPD patients and control cases through western blotting. Quantitative analysis of scanning densitometry was performed. As shown in Figure 1C and $1 \mathrm{D}$, the expression of pulmonary DJ-1 was obviously down-regulated in COPD patients compared with control subjects.
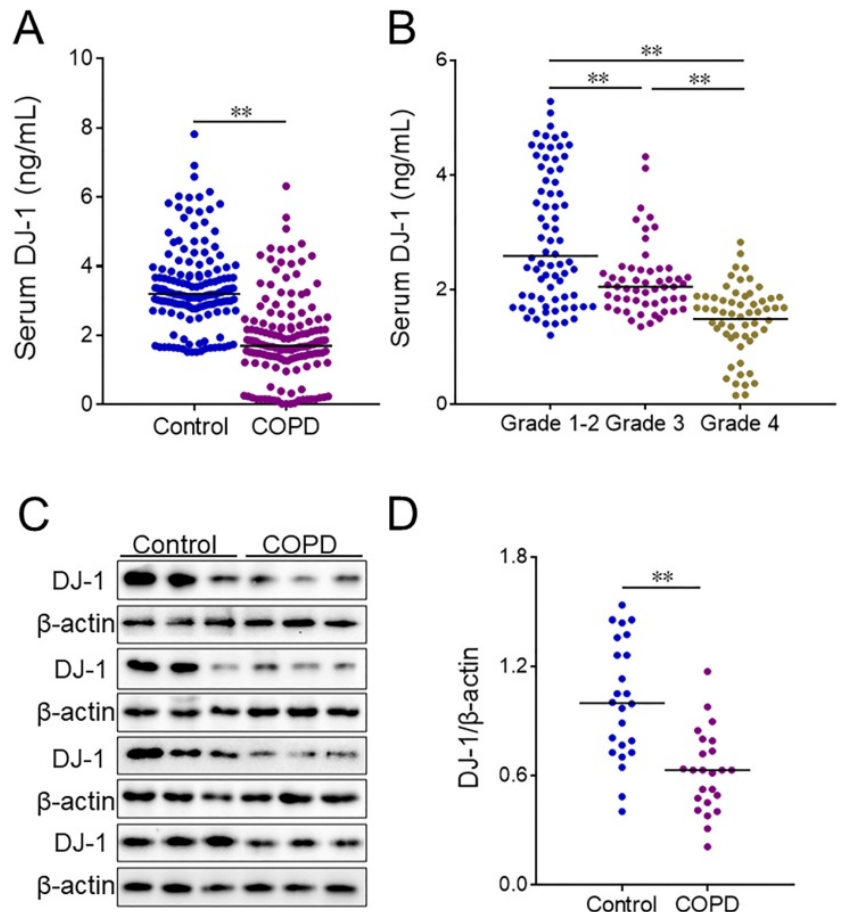

Figure 1. The level of DJ-1 between COPD patients and control subjects. Serum and lung tissues were collected from COPD patients and control subjects. The level of DJ-1 was detected in serum using ELISA. (A) The level of serum DJ-1 was detected between COPD patients and control subjects. (B) The level of serum DJ-1 was evaluated in different grades of COPD patients. (C, D) The level of DJ-1 was measured in lung tissues using western blotting. (C) The protein expression of DJ-1 was evaluated in lung tissues between COPD patients and control subjects. (D) Quantitative analysis of scanning densitometry was performed. All data were represented as means \pm S.E.M. $(\mathrm{N}=24)$. ${ }^{*} \mathrm{*}<<0.01$.

Table 1. Demographic and biochemical characteristics between COPD patients and control subjects

\begin{tabular}{llll}
\hline Variables & COPD $(\mathrm{n}=180)$ & Control $(\mathrm{n}=180)$ & $P$ \\
\hline Age $($ years $)$ & $74.0(68.5,82.0)$ & $67.0(50.3,79.6)$ & 0.437 \\
Male, $\mathrm{n}(\%)$ & $132(73.2)$ & $114(63.2)$ & 0.351 \\
Hospital stay (day) & $10.0(7.0,14.0)$ & N.S. & N.S. \\
WBC $\left(10^{9} / \mathrm{L}\right)$ & $6.79(5.07,9.52)$ & $5.32(4.32,6.93)$ & $<0.01$ \\
Neutrophils $\left(10^{9} / \mathrm{L}\right)$ & $4.74(3.38,7.22)$ & $3.15(2.21,3.96)$ & $<0.01$ \\
Lymphocyte $\left(10^{9} / \mathrm{L}\right)$ & $1.08(0.78,1.45)$ & $2.15(1.72,2.63)$ & $<0.01$ \\
Monocyte $\left(10^{9} / \mathrm{L}\right)$ & $0.47(0.33,0.68)$ & $0.35(0.29,0.44)$ & $<0.05$ \\
CRP $(\mu \mathrm{mg} / \mathrm{mL})$ & $88.3 \pm 9.6$ & $36.5 \pm 7.4$ & $<0.01$ \\
TNF- $\mathrm{(ng} / \mathrm{mL})$ & $103.5 \pm 7.9$ & $30.3 \pm 6.2$ & $<0.01$ \\
IL-6 $(\mathrm{pg} / \mathrm{mL})$ & $48.6 \pm 10.6$ & $19.6 \pm 7.6$ & $<0.05$ \\
MCP-1 $(\mathrm{pg} / \mathrm{mL})$ & $206.5 \pm 42.5$ & $58.6 \pm 7.6$ & $<0.01$ \\
FVC $(\mathrm{L})$ & $1.88(1.36,2.47)$ & N.S. & N.S. \\
FEV1 $(\%)$ & $40.4(27.6,64.8)$ & N.S. & N.S. \\
FEV1/FVC $(\%)$ & $51.3(43.3,58.5)$ & N.S. & N.S. \\
\hline
\end{tabular}


A

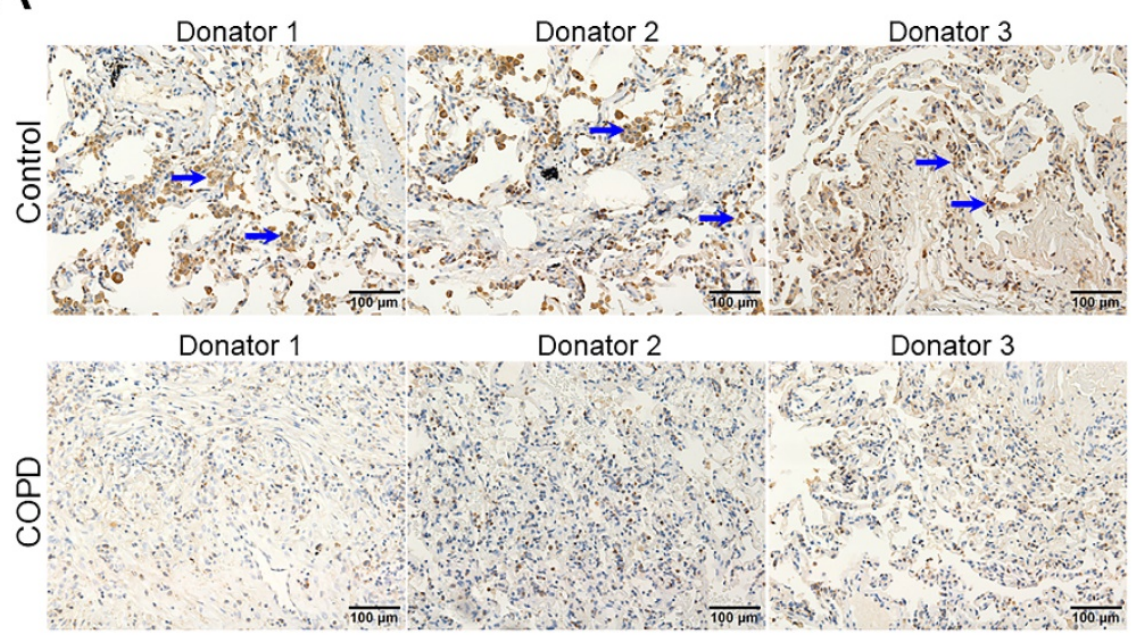

B
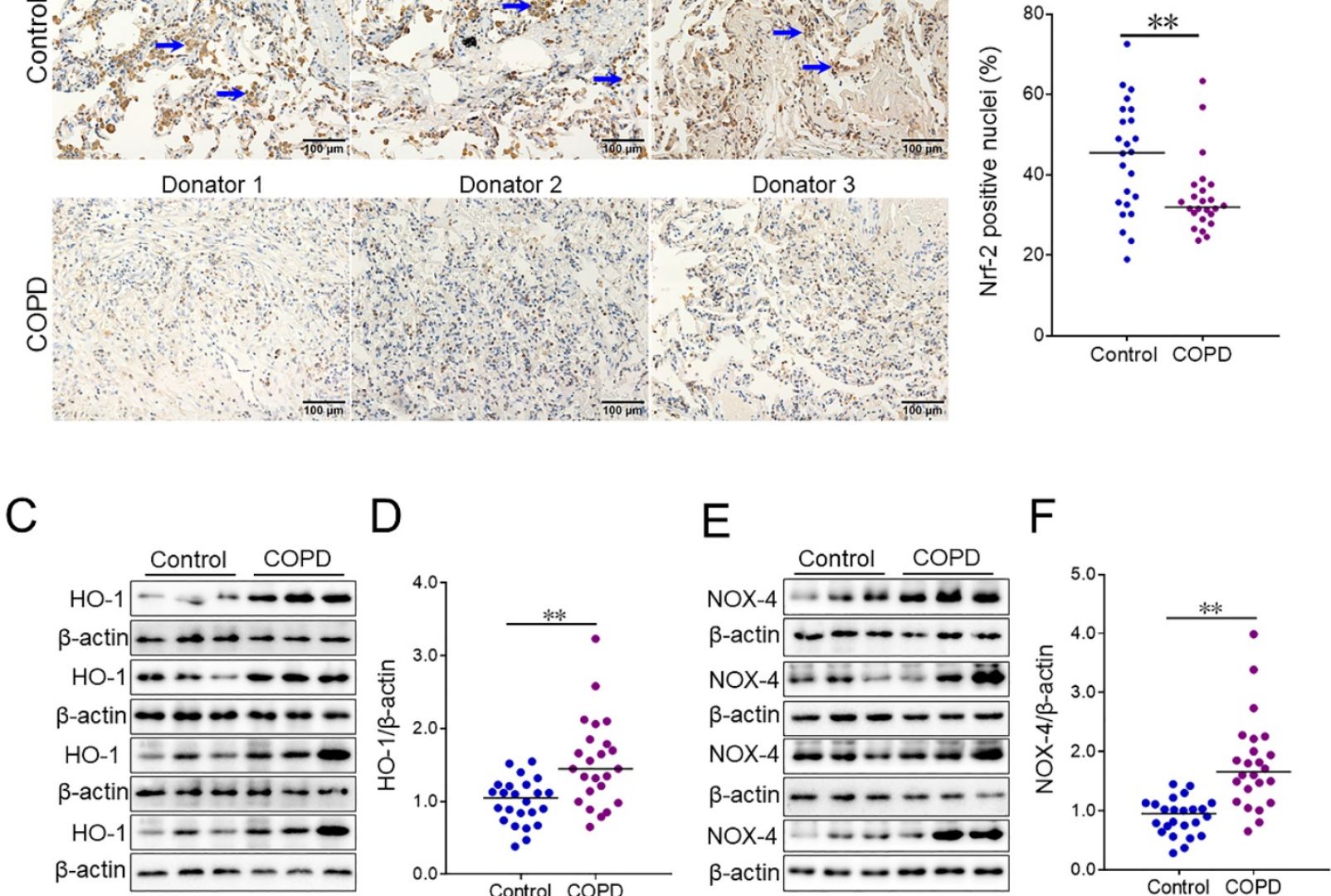

Figure 2. The levels of oxidative stress markers between COPD patients and control subjects. (A, B) Pulmonary Nrf-2-positive nuclei were measured using IHC. (A) Representative field. Blue arrows indicate Nrf-2-positive nuclei. Original magnification: $\times 400$. (B) Quantitative analysis of positive nuclei was performed. (C-F) Pulmonary HO-1 and NOX-4 were detected between COPD patients and control subjects through western blotting. (C) Representative bands of HO-1. (E) Representative bands of NOX-4. (D, F) Quantitative analysis of scanning densitometry was performed. All data were represented as means \pm S.E.M. $(N=24)$. $* * P<0.01$.

\section{Pulmonary markers of oxidative stress in COPD patients and control subjects}

The level of pulmonary Nrf-2 was detected using IHC. The numbers of pulmonary Nrf-2-positive nuclei were compared in COPD patients and control subjects. As shown in Figure 2A and 2B, the number of pulmonary Nrf-2-positive nuclei was decreased in COPD patients compared with control subjects. Additionally, the markers of oxidative stress were measured in lung tissues of COPD patients and control subjects. As shown in Figure 2C-2F, the expression of pulmonary heme oxygenase-1 (HO-1) and NADPH oxidase 4 (NOX-4) was increased in COPD patients.

\section{Pulmonary VDR-positive nuclei in COPD patients and control subjects}

VDR-positive nuclei were analyzed in lung tissues between COPD patients and control subjects using IHC. As shown in Figure $3 \mathrm{~A}$ and $3 \mathrm{~B}$, the number of pulmonary VDR-positive nuclei was less in COPD patients than these in control subjects.

\section{Correlations of pulmonary function with pulmonary DJ-1, Nrf-2 and VDR in COPD patients}

The correlations of FEV1 with pulmonary DJ-1, Nrf-2 and VDR were analyzed. As expected, there was an obviously positive correlation between FEV1 and pulmonary DJ-1 expression in COPD patients $(r=0.632, P<0.01)$ (Figure 4A). Moreover, we found that pulmonary DJ-1 expression was significantly associated with Nrf-2- and VDR-positive nuclei in lung tissues of COPD patients $(r=0.521, \quad P<0.01$; $r=0.527, P<0.01$ ) (Figure $4 \mathrm{~B}$ and $4 \mathrm{C}$ ). In addition, the associations of DJ-1 with Nrf-2 and VDR were evaluated in lung tissues of COPD patients. As shown in Figure $4 \mathrm{D}$ and $4 \mathrm{E}, \mathrm{DJ}-1$ expression was notably correlated with Nrf-2- and VDR-positive nuclei in lung tissues of COPD patients $(r=0.418, \quad P<0.05$; $r=0.436, P<0.01)$. Moreover, the association between 
VDR-positive nuclei and Nrf-2-positive nuclei was analyzed. As shown in Figure $4 \mathrm{~F}$, there was an obvious positive correlation between VDR-positive nuclei and Nrf-2-positive nuclei in lung tissues of COPD patients $(r=0.425, P<0.05)$.

\section{Discussion}

In the current research, we mainly detected the levels of DJ-1, VDR, Nrf-2 and related markers of oxidative stress between COPD patients and control subjects. The associations of FEV1 and DJ-1, VDR and Nrf-2 were evaluated in COPD patients. The present research mostly found that: (1) Pulmonary and serum DJ-1 was decreased in COPD patients; (2) Pulmonary VDR and Nrf-2 were reduced in COPD patients; (3) Pulmonary HO-1 and NOX-4 were elevated in COPD patients; (4) There were positive correlations among pulmonary function, DJ-1, VDR and Nrf-2 in lung tissues of COPD patients.

The pathogenesis and pathology of COPD are complex, but excessive oxidative stress is an important cause and mechanism of COPD [2]. Compelling data suggest that oxidative stress derived from ROS present in COPD patients and evoked

A

Donator 1 Donator 2 Donator 3

B
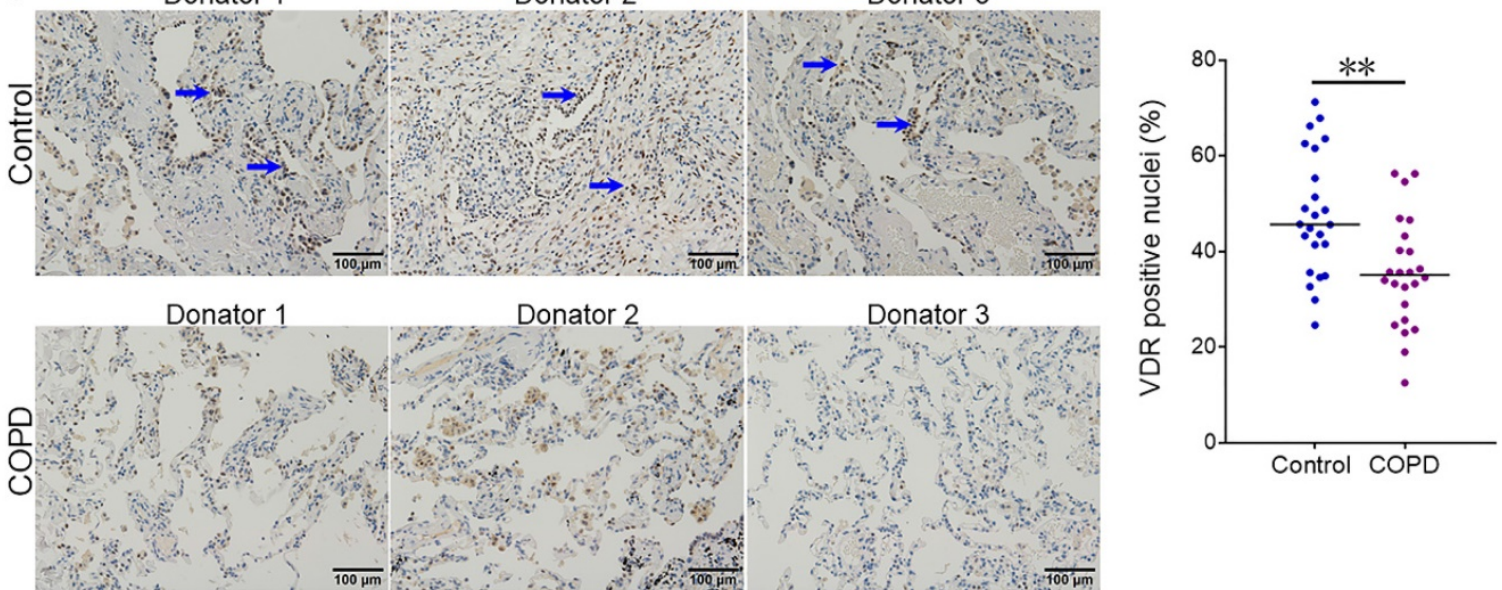

Figure 3. The level of pulmonary VDR-positive nuclei between COPD patients and control subjects. (A) Pulmonary VDR-positive nuclei were measured using IHC. Blue arrows indicate VDR-Y-positive nuclei. Original magnification: $\times 400$. (B) Quantitative analysis of positive nuclei was performed. All data were represented as means \pm S.E.M. $(\mathrm{N}=24) . * * P<0.01$.

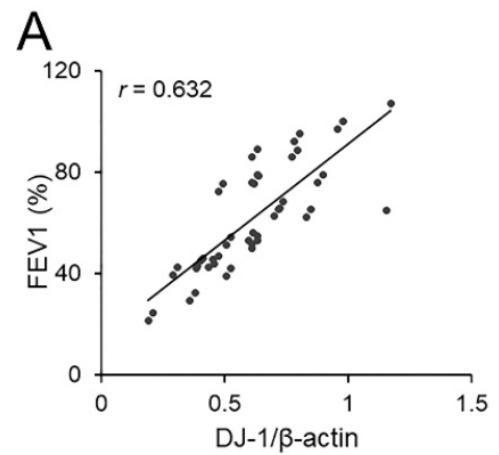

B

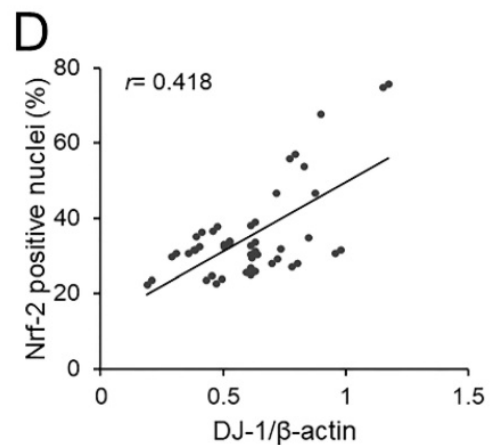

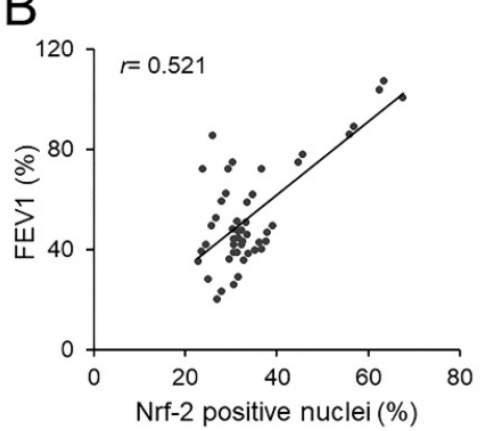

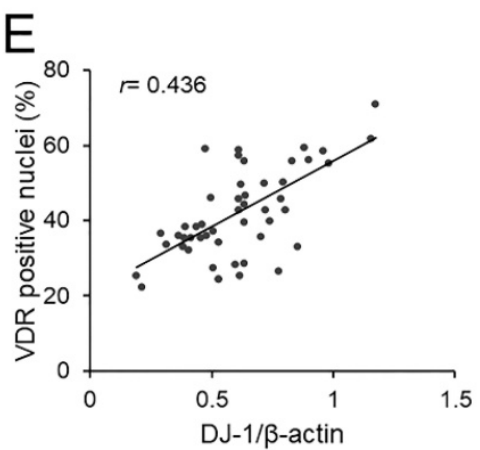

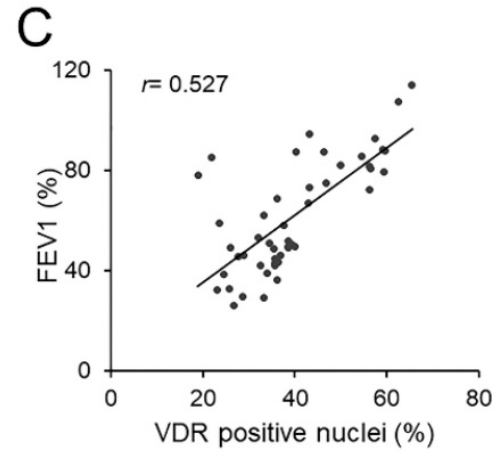

F

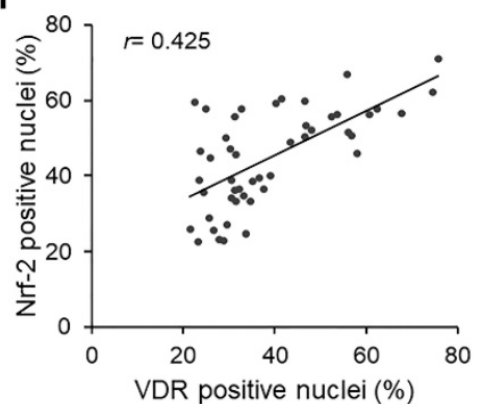

Figure 4. Associations among FEV1, DJ-1, Nrf-2 and VDR in COPD patients. (A-C) Associations of FEV1 and pulmonary DJ-1, VDR and Nrf-2 were analyzed in COPD patients. (A) FEV1 vs DJ-1. (B) FEV1 vs Nrf-2. (C) FEV1 vs VDR. (D-F) Associations among DJ-1, VDR and Nrf-2 in lung tissues of COPD patients. (D) Nrf-2 vs DJ-1. (E) VDR vs DJ-1. (F) VDR vs Nrf-2. 
several inflammatory and immune stimuli in epithelial cells of the airways [21]. Recently, a research found that the markers of oxidative stress were elevated in peripheral blood mononuclear cells derived from COPD patients [22]. To our knowledge, Nrf-2 is a key transcription factor that regulates the downstream antioxidant defense system and exerts a significant function in COPD [23]. The previous in vivo experiment found that cigarette smoking, the primary cause of COPD, evoked ROS excessive production and Nrf-2 downregulation in the human lung alveolar epithelium cell line [24]. Meanwhile, our results indicated HO-1 and NOX-4 were increased in lungs of COPD patients. On the contrary, pulmonary Nrf-2-positive nuclei was reduced in COPD patients.

It is commonly understood that HO- 1 is the important downstream molecule of Nrf-2. However, NOX-4 have been proved to be the main source of ROS under various pathological conditions. Cigarette smoking is the most important risk factor for COPD $[3,4]$. Mounting evidences proved that cigarette smoking induced the production of ROS and evoked oxidative stress [25]. Simultaneously, cigarette smoking elevated the expression and activity of HO-1 in mouse cerebral vascular endothelial cells [26]. When human bodies suffer from ROS attack, the bodies may activate the anti-oxidization system in the body to eliminate ROS. It is quite possible that these two molecules of opposite functions showed similar expression in human bodies. HO-1 is mainly induced under several stimuli such as hypoxia, oxidative stress, cytokines, cigarette smoke and heavy metals in biological systems [27]. Different intracellular signaling molecules and transcription factors are associated with HO-1 expressions such as Nrf-2, Tramtrack and Broad complex (BTB), activator protein-1 (AP-1), nuclear factor kappa-B (NF-kB) and Mitogen-activated protein kinase (MAPK) [28]. Though cigarette smoking inhibited Nrf-2 nuclear translocation, it also activated several inflammatory signaling, such as AP-1, NF- $\mathrm{kB}$ and MAPKs, and then induced oxidative stress $[29,30]$. An earlier study found that pulmonary Nrf-2 was reduced in COPD patients [31]. However, recent researches indicated HO- 1 was increased in COPD patients $[32,33]$. The human body is an organic and complex whole. Intracellular redox imbalance results in different trends of Nrf-2 and HO-1 in human bodies. Maybe those reasons partially explain why $\mathrm{Nrf}-2$ is decreased, HO- 1 and NOX-4 are increased in COPD patients.

DJ-1 is a redox sensitive molecular and stabilizer of Nrf-2 [10]. Several researches indicated that DJ-1 exerted important roles in the defense against oxidative stress. The data reveal that DJ-1 inhibited
Nrf2 from combining with Keap-1 and attenuated the subsequent ubiquitination of Nrf-2, which was essential for maintaining the stability of the antioxidant enzymes under ROS stimulation $[12,13]$. In the absence of oxidative stress, DJ-1-defcient mice showed more severe myocardial injury in response to ischemia [34]. In the Parkinson's disease, oxidative stress mediated reduction in DJ-1 enhances CML a-synuclein in cells [35]. Simultaneously, the level of DJ-1 was detected in serum and lung tissues. Our results suggested that the level of DJ-1 was decreased in serum and lung tissues of COPD patients. Moreover, the levels of DJ-1 were further analyzed in different grades of COPD patients. The results found that serum DJ-1 was gradually decreased in parallel with pulmonary function decline in COPD patients. Not only that, correlation analysis indicated that the expression of DJ-1 was positively associated with Nrf-2-positive nuclei in lung tissues of COPD patients. These results provide evidences that DJ-1 reduction is correlated with the downregulation of Nrf-2 in lung tissues of COPD patients.

VDR was the nuclear receptor for the biologically most active vitamin $\mathrm{D}$ metabolite 1a,25-dihydroxyvitamin D3, which not only exerted a significant role in the metabolism and homeostasis of calcium and phosphate, but also involved in the process of cellular proliferation and differentiation, anti-inflammatory and anti-oxidative function [14, 35-38]. Increasing evidences indicated that VDR played an important function in the process of COPD during the past few decades [39]. Recently, in vivo and in vitro experiments indicated that vitamin $\mathrm{D}$ supplementation could activate VDR and upregulate Nrf-2 expression in different types cells [15-17]. Vitamin D deficiency in obese rats exacerbated nonalcoholic fatty liver disease and elevated oxidative stress marker in liver [40]. This data suggested Nrf-2 may be the downstream protein of VDR. In the current study, we found that VDR- and Nrf-2-positive nuclei were decreased in lung tissues of COPD patients. In addition, we found that VDR was positively associated with DJ-1 and Nrf-2 in lung tissues of COPD patients. These results indicated DJ-1 reduction and VDR downregulation may together contribute to pulmonary Nrf-2 degression. Pulmonary Nrf-2 downregulation further resulted in the insufficiency of antioxidant defense system, damaged pulmonary epithelial cells and finally evoked the occurrence and development of COPD. These results provide a possible mechanistic explanation by which the downregulation of DJ-1 and VDR cause pulmonary function decline in COPD patients.

There are a few defects in the current study. Firstly, this was only a case-control study, so the 
causal relationship between pulmonary Nrf-2 reduction and COPD was unclear, further animal experiments in vivo would help resolve this confusion. Secondly, whether DJ-1 and VDR downregulation associating with Nrf-2 reduction involved in COPD needs to further confirm in vitro and in vivo experimental studies. Thirdly, the mechanism of reduction of Nrf-2 and elevation of Nrf-2' downstream target protein were obscure in COPD patients. In vivo and in vitro experiments may demonstrate the mechanism. Fourthly, this was a single-center and small sample size study, a larger sample size and multicenter study is required in the future research.

\section{Conclusions}

In summary, this study mainly analyzed the correlations among pulmonary function, DJ-1, VDR and Nrf-2 in COPD patients in a hospital-based case-control study. Our results demonstrate that pulmonary and serum DJ-1 is decreased in COPD patients. Meanwhile, pulmonary VDR and Nrf-2 are reduced in COPD patients. Correlation analysis suggested that pulmonary DJ-1, VDR and Nrf-2 is positively associated with FEV1 in COPD patients. In addition, there are positive correlations among DJ-1, VDR and Nrf-2 in lung tissues of COPD patients.

\section{Acknowledgements}

Thanks to the physical examination center of Second Affiliated Hospital for the assistance from all the staff.

\section{Funding Sources}

This study was supported by National Natural Science Foundation of China (91743105 and 81630084) and National Natural Science Foundation Incubation Program of the Second Affiliated Hospital of Anhui Medical University (2020GQFY05).

\section{Statement of Ethics}

Ethics Committee of Anhui Medical University approved this study.

\section{Authors' contributions}

YX and LF designed the study and undertook most of the work, they should be regarded as co-first author. HXX, LZ, ZXT, LXW, WC, DXX and HZ participated in data collection and analysis. All authors have contributed to the last version of the manuscript. The authors read and approved the final manuscript.

\section{Competing Interests}

The authors have declared that no competing interest exists.

\section{References}

1. Vestbo J, Hurd SS, Agustí AG, Jones PW, Vogelmeier C, Anzueto A, et al. Global strategy for the diagnosis, management, and prevention of chronic obstructive pulmonary disease: GOLD executive summary. Am J Respir Crit Care Med. 2013; 1874: 347-65.

2. Vijayan VK. Chronic obstructive pulmonary disease. Indian J Med Res. 2013; 1372: 251-69.

3. Rabe KF, Watz H. Chronic obstructive pulmonary disease. Lancet. 2017; 389: 1931-40.

4. Lareau SC, Fahy B, Meek P, Wang A. Chronic Obstructive Pulmonary Disease (COPD). Am J Respir Crit Care Med. 2019; 199: P1-2.

5. Nagakubo D, Taira T, Kitaura H, Ikeda M, Tamai K, Iguchi-Ariga SM, et al. DJ-1, a novel oncogene which transforms mouse NIH3T3 cells in cooperation with ras. Biochem Biophys Res Commun. 1997; 231: 509-13.

6. Bonifati V, Rizzu P, van Baren MJ, Schaap O, Breedveld GJ, Krieger E, et al. Mutations in the DJ-1 gene associated with autosomal recessive early-onset parkinsonism. Science. 2003; 299: 256-9.

7. Kinumi T, Kimata J, Taira T, Ariga H, Niki E. Cysteine-106 of DJ-1 is the most sensitive cysteine residue to hydrogen peroxide-mediated oxidation in vivo in human umbilical vein endothelial cells. Biochem Biophys Res Commun. 2004; 317: 722-8.

8. Yanagisawa D, Kitamura $\mathrm{Y}$, Inden M, Takata K, Taniguchi T, Morikawa S, Morita $\mathrm{M}$, et al. DJ-1 protects against neurodegeneration caused by focal cerebral ischemia and reperfusion in rats. J Cereb Blood Flow Metab. 2008; 28 : 563-78.

9. Takahashi-Niki K, Kato-Ose I, Murata H, Maita H, Iguchi-Ariga SM, Ariga H. Epidermal Growth Factor-dependent Activation of the Extracellular Signal-regulated Kinase Pathway by DJ-1 Protein through Its Direct Binding to c-Raf Protein. J Biol Chem. 2015; 290: 17838-47.

10. Billia F, Hauck L, Grothe D, Konecny F, Rao V, Kim RH, et al. Parkinsonsusceptibility gene DJ-1/PARK7 protects the murine heart from oxidative damage in vivo. Proc Natl Acad Sci U S A. 2013; 110: 6085-90.

11. Liu R, Yan X. Sulforaphane protects rabbit corneas against oxidative stress injury in keratoconus through activation of the Nrf-2/HO-1 antioxidant pathway. Int J Mol Med. 2018; 42: 2315-28.

12. Lee E, Yin Z, Sidoryk-Węgrzynowicz M, Jiang H, Aschner M. 15-Deoxy$\Delta 12,14$-prostaglandin $\mathrm{J}_{2}$ modulates manganese-induced activation of the NF-KB, Nrf2, and PI3K pathways in astrocytes. Free Radic Biol Med. 2012; 52: 1067-74.

13. Cuevas S, Yang Y, Konkalmatt P, Asico LD, Feranil J, Jones J, et al. Role of nuclear factor erythroid 2-related factor 2 in the oxidative stress-dependent hypertension associated with the depletion of DJ-1. Hypertension. 2015; 65: 1251-7.

14. Fathi N, Ahmadian E, Shahi S, Roshangar L, Khan H, Kouhsoltani M, et al. Role of vitamin D and vitamin D receptor (VDR) in oral cancer. Biomed Pharmacother. 2019; 109: 391-401.

15. Chen L, Yang R, Qiao W, Zhang W, Chen J, Mao L, et al. 1,25-Dihydroxyvitamin $\mathrm{D}$ exerts an antiaging role by activation of Nrf2-antioxidant signaling and inactivation of p16/p53-senescence signaling. Aging Cell. 2019; 18: e12951.

16. Dai Y, Zhang J, Xiang J, Li Y, Wu D, Xu J. Calcitriol inhibits ROS-NLRP3-IL-1 $\beta$ signaling axis via activation of Nrf2-antioxidant signaling in hyperosmotic stress stimulated human corneal epithelial cells. Redox Biol. 2019; 21: 101093.

17. Teixeira TM, da Costa DC, Resende AC, Soulage CO, Bezerra FF, Daleprane JB. Activation of Nrf2-Antioxidant Signaling by 1,25-Dihydroxycholecalciferol Prevents Leptin-Induced Oxidative Stress and Inflammation in Human Endothelial Cells. J Nutr. 2017; 147: 506-13.

18. Fu L, Chen YH, Xu S, Yu Z, Zhang ZH, Zhang C, et al. Oral cholecalciferol supplementation alleviates lipopolysaccharide-induced preterm delivery partially through regulating placental steroid hormones and prostaglandins in mice. Int Immunopharmacol. 2019; 69: 235-44.

19. Fu L, Chen $\mathrm{YH}$, Bo QL, Song $\mathrm{YP}, \mathrm{Ma} \mathrm{L}$, Wang B, et al. Lipopolysaccharide Downregulates $11 \beta$-Hydroxysteroid Dehydrogenase 2 Expression through Inhibiting Peroxisome Proliferator-Activated Receptor- $\gamma$ in Placental Trophoblasts. J Immunol. 2019; 203: 1198-207.

20. Fei J, Fu L, Hu B, Chen YH, Zhao H, Xu DX, et al. Obeticholic acid alleviate lipopolysaccharide-induced acute lung injury via its anti-inflammatory effects in mice. Int Immunopharmacol. 2019; 66: 177-84.

21. Zuo L, He F, Sergakis GG, Koozehchian MS, Stimpfl JN, Rong Y, et al. Interrelated role of cigarette smoking, oxidative stress, and immune response in COPD and corresponding treatments. Am J Physiol Lung Cell Mol Physiol. 2014; 307: L205-18.

22. Fratta Pasini AM, Stranieri C, Ferrari M, Garbin U, Cazzoletti L, Mozzini C, et al. Oxidative stress and Nrf2 expression in peripheral blood mononuclear cells derived from COPD patients: an observational longitudinal study. Respir Res. 2020 Jan; 21: 37. 
23. Mizumura K, Maruoka S, Shimizu T, Gon Y. Role of Nrf2 in the pathogenesis of respiratory diseases. Respir Investig. 2020; 58: 28-35.

24. Mahalanobish S, Dutta S, Saha S, Sil PC. Melatonin induced suppression of ER stress and mitochondrial dysfunction inhibited NLRP3 inflammasome activation in COPD mice. Food Chem Toxicol. 2020; 144: 111588.

25. Pan M, Zheng Z, Chen Y, Sun N, Zheng B, Yang Q, et al. Angiotensin-(1-7) Attenuated Cigarette Smoking-related Pulmonary Fibrosis via Improving the Impaired Autophagy Caused by Nicotinamide Adenine Dinucleotide Phosphate Reduced Oxidase 4-Dependent Reactive Oxygen Species. Am J Respir Cell Mol Biol. 2018; 59(3): 306-19.

26. Shih RH, Lee IT, Hsieh HL, Kou YR, Yang CM. Cigarette smoke extract induces HO-1 expression in mouse cerebral vascular endothelial cells: involvement of c-Src/NADPH oxidase/PDGFR/JAK2/STAT3 pathway. J Cell Physiol. 2010; 225(3): 741-50.

27. Waza AA, Hamid Z, Ali S, Bhat SA, Bhat MA. A review on heme oxygenase-1 induction: is it a necessary evil. Inflamm Res. 2018; 67(7): 579-88.

28. Farombi EO, Surh YJ. Heme oxygenase- 1 as a potential therapeutic target for hepatoprotection. J Biochem Mol Biol. 2006; 39(5): 479-91.

29. Fu L, Fei J, Tan ZX, Chen YH, Hu B, Xiang HX, et al. Low Vitamin D Status Is Associated with Inflammation in Patients with Chronic Obstructive Pulmonary Disease. J Immunol. 2021; 206(3): 515-23.

30. Kaur G, Bagam P, Pinkston R, Singh DP, Batra S. Cigarette smoke-induced inflammation: NLRP10-mediated mechanisms. Toxicology. 2018; 398-399: $52-67$

31. Sun $X$, Chen L, He Z. PI3K/Akt-Nrf2 and Anti-Inflammation Effect of Macrolides in Chronic Obstructive Pulmonary Disease. Curr Drug Metab. $2019 ; 20(4)$ : 301-4.

32. Fratta Pasini AM, Stranieri C, Ferrari M, Garbin U, Cazzoletti L, Mozzini C, et al. Oxidative stress and Nrf2 expression in peripheral blood mononuclear cells derived from COPD patients: an observational longitudinal study. Respir Res. 2020; 21(1): 37 .

33. Maestrelli P, El Messlemani AH, De Fina O, Nowicki Y, Saetta M, Mapp C, Fabbri LM. Increased expression of heme oxygenase (HO)-1 in alveolar spaces and HO-2 in alveolar walls of smokers. Am J Respir Crit Care Med. 2001 Oct 15;164(8 Pt 1):1508-13.

34. Dongworth RK, Mukherjee UA, Hall AR, Astin R, Ong SB, Yao Z, et al. DJ-1 protects against cell death following acute cardiac ischemia-reperfusion injury. Cell Death Dis. 2014; 5: e1082.

35. Sharma N, Rao SP, Kalivendi SV. The deglycase activity of DJ-1 mitigates a-synuclein glycation and aggregation in dopaminergic cells: Role of oxidative stress mediated downregulation of DJ-1 in Parkinson's disease. Free Radic Biol. Med. 2019; 135: 28-37.

36. Zhang M, Lin L, Xu C, Chai D, Peng F, Lin J. VDR Agonist Prevents Diabetic Endothelial Dysfunction through Inhibition of Prolyl Isomerase-1-Mediated Mitochondrial Oxidative Stress and Inflammation. Oxid Med Cell Longev. 2018; 2018: 1714896

37. Bandera Merchan B, Morcillo S, Martin-Nuñez G, Tinahones FJ, MacíasGonzález M. The role of vitamin D and VDR in carcinogenesis: Through epidemiology and basic sciences. J Steroid Biochem Mol Biol. 2017; 167: 203-18.

38. Cantorna MT, Snyder L, Lin YD, Yang L. Vitamin D and 1,25(OH)2D regulation of T cells. Nutrients. 2015; 7: 3011-21.

39. Fei J, Fu L, Cao W, Hu B, Zhao H, Li JB. Low Vitamin D Status Is Associated with Epithelial-Mesenchymal Transition in Patients with Chronic Obstructive Pulmonary Disease. J Immunol. 2019; 203: 1428-35.

40. Roth CL, Elfers CT, Figlewicz DP, Melhorn SJ, Morton GJ, Hoofnagle A, et al. Vitamin $\mathrm{D}$ deficiency in obese rats exacerbates nonalcoholic fatty liver disease and increases hepatic resistin and Toll-like receptor activation. Hepatology. 2012; 55: 1103-11. 